\title{
Caspase-9 was involved in cell apoptosis in human dental pulp stem cells from deciduous teeth
}

\author{
HONG QIAN $^{1}$, QUN HUANG ${ }^{2}$, YU-XIANG CHEN ${ }^{2}$, QIONG LIU ${ }^{1}$, JING-XIAN FANG ${ }^{1}$ and MAN-WEN YE ${ }^{2}$ \\ ${ }^{1}$ Department of Pediatric Dentistry, Stomatological Hospital, Southern Medical University, Guangzhou, Guangdong 510280; \\ ${ }^{2}$ Department of Dentistry, Guangdong Women and Children Hospital, Guangzhou, Guangdong 511442, P.R. China
}

Received September 26, 2017; Accepted March 22, 2018

DOI: $10.3892 / \mathrm{mmr} .2018 .9046$

\begin{abstract}
As one type of adult stem cells (ASCs), human dental pulp stem cells (HDPSCs) have several properties, including high proliferation rate, self-renewal capability, and multi-lineage differentiation. However, the apoptotic mechanism underlying the development of dental pulp cells remains unclear. In the present study, a significant increase of apoptosis was observed in HDPSCs from the deciduous teeth compared with that from adult permanent teeth. In addition, the occurrence of cytochrome $c$ expression and mitochondrial-mediated apoptosis pathway activity in HDPSCs were confirmed by quantitative polymerase chain reaction, and western blotting. Although caspase- 8 and caspase- 9 showed higher expression in deciduous teeth than in adult permanent teeth, only the knockdown of caspase-9 via RNA interference in HDPSC cells exhibited a significant reduction in apoptosis, and caspase-3 expression and activity. All these results revealed that caspase- 9 and activated caspase- 3 predominantly regulates cell apoptosis in HDPSCs from deciduous teeth.
\end{abstract}

\section{Introduction}

During the past decades, adult stem cells (ASCs) have been separated from different tissues. As one type of ASCs, human dental pulp stem cells (HDPSCs) isolated from human dental pulp in adult permanent and exfoliated deciduous teeth (SHED) $(1,2)$ have the capability of differentiation into osteoblasts, odontoblasts, adipocytes and neuronal-like cells (3). Strikingly, HDPSCs can regenerate a dentin-pulp-like complex in normal human teeth (4), showing that HDPSCs represent a novel ASC population (2).

In spite of the great potential in tissue engineering $(5,6)$, the molecular mechanisms underlying cell apoptosis in HDPSCs

Correspondence to: Dr Hong Qian, Department of Pediatric Dentistry, Stomatological Hospital, Southern Medical University, 366 Jiangnan Avenue South, Guangzhou, Guangdong 510280, P.R. China

E-mail: sqianh2@126.com

Key words: human dental pulp stem cells, apoptosis, caspase-9, RNA interference remain unclear. Interestingly, there are very few data on the occurrence of apoptosis in the cells of the dental pulp (7). A disintegrin and metalloproteinase 28 (ADAM28) was reported to be involved in the proliferation, differentiation, and apoptosis of HDPSCs (8), but a prior study by Muthna et al reported that the irradiation of adult HDPSCs provokes activation p53, cell cycle arrest and senescence instead of apoptosis (3). So far, few evidences showed the occurrence of apoptosis in HDPSCs.

As reviewed previously (9-11), three typical pathways, including extrinsic pathway, intrinsic pathway and a perforin/granzyme pathway, were involved in cell apoptosis. Each of three pathways requires activation of caspase-8, caspase-9 and caspase-10, which in turn activate caspase-3. However, whether HDPSCs contain the same pathways remains unknown.

Here, we checked the existences of the extrinsic and intrinsic pathways of apoptosis in HDPSCs isolated from adult permanent teeth and deciduous teeth by RNA interference (RNAi), RT-qPCR and other experiments. RNAi has been widely accepted as an excellent system for the targeted silencing of gene expression, in which activation of small-interfering RNA [(siRNA), 21 nucleotides] pathway results in the degradation of a specific targeted mRNA $(12,13)$. The specificity and potency of siRNA in cell culture and in animal studies has suggested that it can be a powerful therapeutic agent. In present study, we knocked down the expressions of caspase- 8 and caspase- 9 by RNAi experiments, respectively, and observed significant reduction of HDPSCs apoptosis in the caspase-9 RNAi group instead of the caspase-8 RNAi group, showing that differences of functional consequences of caspase- 8 and caspase-9. The caspase- 3 expression and activity assays revealed that caspase- 3 activated by caspase- 9 regulated apoptosis in HDPSCs.

\section{Materials and methods}

Ethics statement. All procedures used in this study conformed to the tenets of the Declaration of Helsinki. The Ethics Committee guidelines of Stomatological Hospital, Southern Medical University approved the protocols used. Informed consent was obtained from all participants.

Subjects and cell culture. Six normal deciduous teeth and 6 adult permanent teeth were collected from 6- to 13-year-old 
children and normal adults, respectively. All the teeth were extracted according to the agreements of patients and regular medical processes. The intact teeth were separated, immersed in a solution of $0.25 \%$ Chloramphenicol solution (30 min), and then kept in PBS solution with Penicillin $(60 \mathrm{mg} / \mathrm{l})$ and Streptomycin $(100 \mathrm{mg} / \mathrm{l})$. The dental pulp was isolated from the teeth, and washed by PBS solutions with Penicillin and Streptomycin, then digested in a solution of $1 \%$ collagenase (Sigma-Aldrich; Merck KGaA, Darmstadt, Germany) for $1 \mathrm{~h}$ at $37^{\circ} \mathrm{C}$. The cells were filtered by 150 mesh nylon, centrifuged $1,000 \mathrm{x} g$ for $5 \mathrm{~min}$, and countered by blood counting chamber. After these processes, the cells were then mixed in DMEM solution (20\% FBS, $100 \mu \mathrm{M}$ ascorbic acid, $2 \mathrm{mM}$ L-glutamine, $60 \mathrm{mg} / 1$ Penicillin and $100 \mathrm{mg} / \mathrm{l}$ Streptomycin). Anti-immunoglobulin M Micro Beads (Miltenyi-Biotec, Bergisch Gladbach, Germany) were used to sort the separated cells, according to the manufacturer's instructions. We then obtained the cell populations that were termed as stem cells from deciduous teeth and adult permanent teeth. These HDPSCs were cultured for further use.

Hematoxylin and Eosin $(H \& E)$ staining. To determine the apoptosis stages of dental pulp cells, H\&E staining was employed to observe the apoptotic processes and stages of stem cells from deciduous teeth and adult permanent teeth. We also used H\&E staining to observe the HDPSC apoptosis processes of RNAi groups and control groups from deciduous teeth.

Apoptosis assay. For Annexin V-FITC apoptosis assay, separated stem cells from deciduous teeth and adult permanent teeth were cultured, trypsinized, washed, and stained with Annexin V/PI apoptosis kit (Multi Sciences Biotech, Dalian, China) in the dark for $15 \mathrm{~min}$ at room temperature. Then, the stained cells were analyzed by flow cytometry.

Reverse transcription-quantitative polymerase chain reaction $(R T-q P C R)$. The mitochondria were extracted by Cell Mitochondria Isolation kit (Beyotime Institute of Biotechnology, Shanghai, China). Using TRIzol (Takara Biotechnology Co., Ltd., Dalian, China), poly-A+ RNA were extracted from the mitochondria, cytoplasm, and whole cells of the HDPSCs from deciduous teeth and adult permanent teeth. RT-qPCR was performed to check the expression of Cytochrome $c$ in these samples. The Cytochrome $c$ primers consisted of the following sequences: (Forward) 5'-CCA ATGAAGATGGGGAGATG-3' and (reverse) 5'-CCGTGA GCAGGGAGAAGAC-3'. The primer pair for the $\beta$-actin housekeeping gene was made up of the following sequences: (Forward) 5'-ATCGTGCGTGACATTAAGGAGAAG-3' and (reverse) 5'-AGGAAGGAAGGCTGGAAGAGTG-3'.

Furthermore, we examined the expression of caspase-9, caspase- 3 and caspase- 8 by RT-qPCR in dental pulp from deciduous teeth and adult permanent teeth. The primers for these three genes were made up of the following sequences: (caspace-9 forward) 5'-AACCCTAGAAAACCTTACCCC-3', (caspace-9 reverse) 5'-CATCACCAAATCCTCCAGAAC-3', (caspase-3 forward) 5'-AGCAAACCTCAGGGAAACATT-3', (caspase-3 reverse) 5'-CTCAGAAGCACACAAACAAAA CT-3', (caspase-8 forward) 5'-GGGAGGAGTTGTGTGGGG TA-3', (caspase-8 reverse) 5'-CAGTCATCGTGGGGCTTGA-3'.
Each sample of cDNA templates $(1 \mu \mathrm{l})$ was added to Bestar $^{\circledR}$ SYBR Green qPCR Master Mix (DBI Bioscience, Shanghai, China). The amplification protocol consisted of a pre-denaturation step at $94^{\circ} \mathrm{C}$ for $2 \mathrm{~min}$, and 40 cycles of the following: A denaturation step at $94^{\circ} \mathrm{C}$ for $20 \mathrm{sec}$, annealing at $58^{\circ} \mathrm{C}$ for $20 \mathrm{sec}$ and extension at $72^{\circ} \mathrm{C}$ for $20 \mathrm{sec}$. Melting curve data were collected to verify PCR specificity. Each mRNA sample was analyzed in triplicate. In addition, the expression was calculated by relative quantification using $\beta$-actin as reference control. Fold expression changes were determined by the $2^{-\Delta \Delta \mathrm{Cq}}$ method (14).

RT-qPCR was performed to check the expression of the RNAi samples of caspase- 8 and caspase- 9 in the HDPSCs from deciduous teeth. The primer sequences of caspase- 8 , caspase- 9 and $\beta$-actin were the same as above.

Western blot. Using lysis buffer (cat. no. P0013) (Beyotime Institute of Biotechnology), total protein was extracted from the mitochondria, cytoplasm, and whole cells of the HDPSCs from deciduous teeth and adult permanent teeth. The protein concentration was determined by BCA assay kit (Thermo Fisher Scientific, Inc., Waltham, MA, USA). $20 \mu \mathrm{g}$ protein was separated by SDS-PAGE (10\% stacking gel and 5\% separation gel) and transferred to a nitrocellulose membrane. The membranes were blocked in 5\% skimmed milk for $1 \mathrm{~h}$ at room temperature, respectively incubated with rabbit anti-human monoclonal antibody against Cytochrome $c$ (dilution, 1:800; cat. no. 4280), mouse anti-human monoclonal antibody against caspase-9 (dilution, 1:2,000; cat. no. 9508), rabbit anti-human monoclonal antibody against caspase-3 (dilution, 1:1,000; cat. no. 14220) and rabbit anti-human monoclonal antibody against caspase-8 (dilution, 1:1,500; cat. no. 4790) (Cell Signaling Technology, Inc., Danvers, MA, USA) at room temperature for $1 \mathrm{~h}$. The membranes were washed with $25 \mathrm{ml}$ TBS containing $0.1 \%$ Tween-20 (TBST) followed by an incubation of secondary antibody (HRP goat anti-rabbit and goat anti-mouse IgG) (dilution, 1:20,000; cat. nos. BA1054 and BA1051; Boster Bioengineering Co., Ltd., Wuhan, China) at room temperature for $40 \mathrm{~min}$. After final washing with $25 \mathrm{ml}$ TBST, the membranes were developed using chemiluminescence and exposed to X-ray films. GAPDH (dilution, 1:10,000; cat. no. RC-5G5; KangChen Bio-tech, Shanghai, China) was used as positive control.

Similarly, we also used western blotting to check the expression of the RNAi samples of caspase- 8 and caspase- 9 in the HDPSCs from deciduous teeth, using GAPDH (RC-5G5) (KangChen Bio-tech) as positive control.

RNAi. RNAi was employed to knock down the expression of caspase- 8 and caspase- 9 in the HDPSCs from deciduous teeth. We designed and chemically synthesized four DNA oligos for each gene and then chose the best one for RT-qPCR assays. The siRNA oligo sequences for caspase- 8 and caspase- 9 were as follows: (caspase-8) siRNA1, 5'-CUACCAGAAAGG UAUACCUTT-3', siRNA2: 5'-GAGGGUCGAUCAUCUAUU ATT-3', siRNA3: 5'-GGGUCGAUCAUCUAUUAAUTT-3', and siRNA4: 5'-GAGCUGCUCUUCCGA AUUATT-3'; (caspase-9) siRNA1: 5'-GAUGCCUGGUUGCUUUAA UTT-3', siRNA2: 5'-CGGUGAAAGGGAUUUAUAATT-3', siRNA3: 5'-CACCCAGUGACAUCUUUGUTT-3', and 
siRNA4: 5'-GCCACUGCCUCAUUAUCAATT-3'. The empty vector, LV3-shRNAs was transfected into HDPSCs, serving as a negative control. All siRNA sequences were examined for specificity using a BLAST search (15) and failed to show homology to any other genes in Homo sapiens.

Both RT-qPCR and western blotting were performed to assay the expression the transfected and untransfected HDPSCs from deciduous teeth. $\beta$-actin and GAPDH were used as internal and positive control in RT-qPCR and Western blot assays, respectively. After investigating the expression, we finally chose the first (caspase- 8 siRNA1) and second (caspase-9 siRNA2) primer of caspase- 8 and caspase-9 for Lentivirus construct (LV3) and following RNAi and RT-qPCR assays, respectively.

Furthermore, the untransfected HDPSCs, HDPSCs transfected with empty vector, caspase- 8 and caspase- 9 siRNAs (caspase-8-siRNA and caspase-9-siRNA), were cultured, trypsinized, washed, and stained with Annexin V-APC/7-AAD apoptosis kit (Multi Sciences Biotech) in the dark for 15 min at room temperature. Then, the stained cells were analyzed by flow cytometry.

Caspase activity assay. Cultured HDPSCs, separated from deciduous teeth, were harvested and lysed with lysis buffer (cat. no. P0013; Beyotime Institute of Biotechnology). After $16,000-20,000 \times \mathrm{g}$ centrifugation at $4^{\circ} \mathrm{C}$ for $10-15 \mathrm{~min}$, the protein concentration was measured using Bradford method. The caspase- 3 activity of untransfected HDPSCs, HDPSCs transfected with empty vector, HDPSCs transfected with caspase- 8 and caspase-9 siRNAs (caspase-8-siRNA and caspase-9-siRNA) were assayed with Caspase-3 Activity Assay kit (Beyotime Institute of Biotechnology) according to manufacturing instruction. In order to check the correlation of caspase-3 expression and activity, RT-qPCR assays were also performed to measure the expression of caspase- 3 in all these four groups of cells.

Statistical analysis. An unpaired Student's t-test and an one-way analysis of variance (ANOVA) in IBM SPSS platform were used to perform statistical analysis. For two groups, unpaired two-tailed Student's t tests were used; for more than two group comparisons, one-way ANOVAs were used followed by the post hoc Tukey's HSD (honest significant difference) test. Statistical significance was determined with unpaired Student's t-test or One-way ANOVA test. $\mathrm{P}<0.05$ was regarded as statistically significant $\left({ }^{*} \mathrm{P}<0.05,{ }^{* *} \mathrm{P}<0.01\right)$. Additionally, Pearson correlation analysis was conducted in Excel to calculate possible correlation between caspase-3 expression and activity.

\section{Results}

Characterization of HDPSCs. The separated cells from deciduous teeth and adult permanent teeth were round or irregular shape (Fig. 1A-D) after primary separation and enrichment by Anti-immunoglobulin M Micro Beads (Miltenyi-Biotec). However, the HDPSCs were fusiform after culture for 2 weeks (Fig. 1E-H). This observation is similar to previous report (16), in which SHED and DPSCs displayed a fibroblastic morphology.
Moreover, in the Annexin V-FITC apoptosis assay, a significant increase of apoptosis was observed in the deciduous teeth than in adult permanent teeth $(\mathrm{P}=0.014<0.05$, unpaired Student's t-test) (Fig. 1I-K).

Expression of Cytochrome c, caspase-9, caspase-8 and caspase-3. In order to find the mechanisms of the different apoptosis level between the HDPSCs from deciduous teeth and adult permanent teeth, we examined the expression of Cytochrome $c$ in the mitochondria of the HDPSCs from deciduous teeth and adult permanent teeth by RT-qPCR and Western blot assay. The results showed that Cytochrome $c$ exhibited a relative higher expression in mitochondria than in cytoplasm of the HDPSCs from adult permanent teeth $(\mathrm{P}<0.01$, unpaired Student's t-test) (Fig. 2A). By contrast, the expression level of Cytochrome $c$ was significantly lower in mitochondria than in cytoplasm of the HDPSCs from deciduous teeth $(\mathrm{P}<0.01$, unpaired Student's t-test) (Fig. 2B). These results were confirmed by Western blot assay, in which the extent of Cytochrome $c$ protein level in mitochondria and cytoplasm of the HDPSCs from adult permanent teeth and deciduous teeth was consistent with that of its transcript level (Fig. 2C).

Furthermore, we measured the expression levels of caspase-9, caspase- 8 and caspase- 3 in the HDPSCs from adult permanent teeth and deciduous teeth, respectively. The RT-qPCR results showed that all three genes had higher transcript level in the HDPSCs from deciduous teeth than in that from adult permanent teeth $(\mathrm{P}<0.01$, unpaired Student's t-test) (Fig. 3A-C). The Western blot assay confirmed the RT-qPCR results, revealing that the protein levels of all three genes were also consistent with their transcript levels (Fig. 3D).

Knock down of caspase-9 induced reduction of apoptosis. In order to knock down the expression of caspase- 8 and caspase- 9 in HDPSCs from deciduous teeth, we designed four primers for each gene. After tests by RT-qPCR and Western blot, the first primer of caspase- 8 (caspase- 8 siRNA1) and the second one of caspase-9 (caspase-9 siRNA2) had greatest reduction of gene expression in both transcript and protein levels $(\mathrm{P}<0.01$, one-way ANOVAs and Tukey's post hoc tests) (Fig. 4A-D). Then, caspase- 8 siRNA1 and caspase- 9 siRNA2 were selected to conduct RNAi experiments. These data revealed efficient knocking down of both genes in HDPSCs (Fig. 4A-D).

After knocking down of caspase- 8 and caspase- 9 by caspase- 8 siRNA1 and caspase- 9 siRNA2, respectively, the protein expression of them were greatly reduced in Western blot assays (Fig. 4E and F).

The Annexin V-FITC apoptosis assay showed caspase-9 had a reduction of apoptosis in the RNAi group (transfected HDPSCs) than in the untransfected HDPSCs $(\mathrm{P}<0.01$, one-way ANOVAs and Tukey's post hoc tests) and in the HDPSCs transfected with empty vector $(\mathrm{P}<0.01$, one-way ANOVAs and Tukey's post hoc tests) (Fig. 5A-E). This observation indicated that caspase- 9 was involved in apoptosis of HDPSCs. By contrast, caspase- 8 showed no significant difference of apoptosis among these groups $(\mathrm{P}>0.05$, one-way ANOVAs and Tukey's post hoc tests) (Fig. 5A-E).

In previous reviews (9-11), caspase-9 was required to activate caspase- 3 and result in cell apoptosis. Whether the expression changes of the upstream genes of caspase- 9 in 

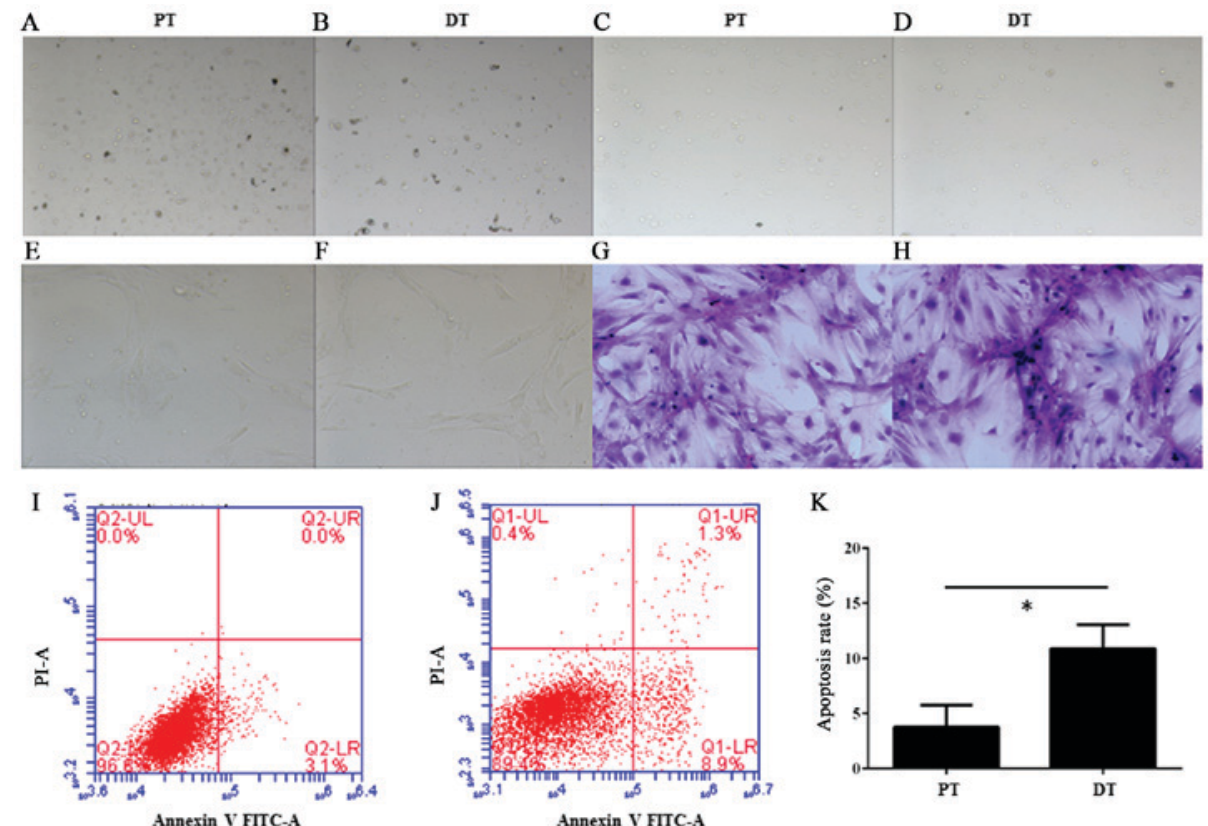

Figure 1. Characterization of cultured human dental pulp stem cells (HDPSCs). (A and B) The cells separated from adult permanent teeth (PT) and deciduous teeth (DT); (C and D) The separated cells enriched by Anti-Immunoglobulin M Micro Beads; (E and F) The HDPSCs after culture for 2 weeks; $(\mathrm{G}$ and $\mathrm{H})$ Hematoxylin and eosin staining of the cultured HDPSCs; (I and J) Annexin V-FITC apoptosis assays of the HDPSCs from (I) adult permanent teeth and $(\mathrm{J})$ deciduous teeth; $(\mathrm{K})$ Apoptosis rates of the HDPSCs from deciduous teeth and adult permanent teeth. Data represent the mean \pm standard deviation $(\mathrm{n}=3)$. Statistical significance was determined with an unpaired Student's t-tests. $\mathrm{P}<0.05$ was regarded as statistically significant $\left({ }^{*} \mathrm{P}<0.05\right)$.
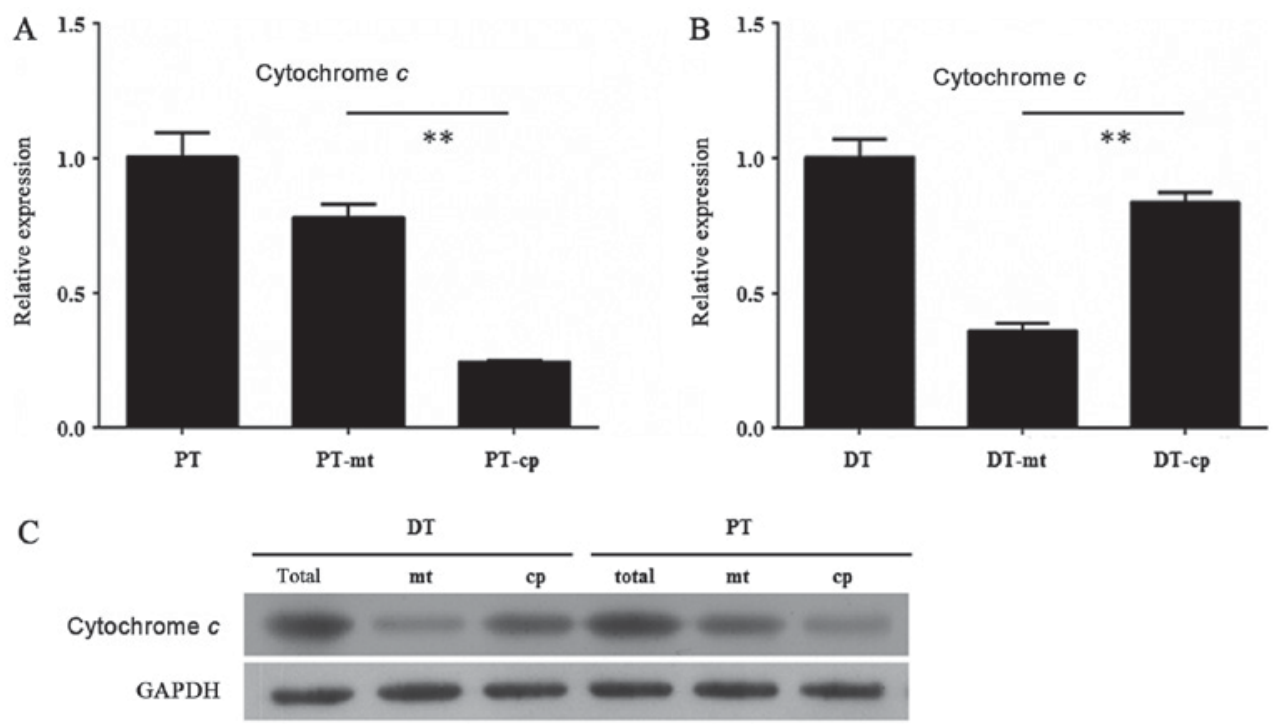

Figure 2. Expression of Cytochrome $c$ in human dental pulp stem cells (HDPSCs). (A) Expression of Cytochrome $c$ in HDPSCs from adult permanent teeth (PT), the mitochondria (mt) and cytoplasm (cp) of HDPSCs from adult permanent teeth; (B) Expression of Cytochrome $c$ in HDPSCs from deciduous teeth (DT), the mt and cp of HDPSCs from deciduous teeth; (C) Western blotting results of Cytochrome $c$ in $\mathrm{mt}$, cp, and whole cells of HDPSCs from adult PT and DT. Data represent the mean \pm standard deviation $(n=3)$. Statistical significance was determined with an unpaired Student's t-tests. $P<0.05$ was regarded as statistically significant $\left({ }^{* *} \mathrm{P}<0.01\right)$.

this pathway contribute to cell apoptosis of HDPSCs remains unclear. Here, we employed RT-qPCR approach to check the expression of four upstream genes (Bax, Bak, Apaf-1 and $c y t C$ ) of caspase-9. The results showed no significant difference of them among the RNAi group, the untransfected HDPSCs and the HDPSCs transfected with empty vector (data not shown here). Therefore, cell apoptosis of HDPSCs ought to be caused by expression changes of caspase- 9 instead of its upstream genes.
Caspase- 3 activity of HDPSCs. The caspase- 3 activity assays showed that HDPSCs transfected with caspase-9 siRNA2 have lower average caspase- 3 activity $(15.79 \pm 6.33 \mathrm{nM} / \mathrm{mg})$ than untransfected HDPSCs $(51.57 \pm 6.55 \mathrm{nM} / \mathrm{mg})$ and HDPSCs transfected with empty vector $(57.81 \pm 10.04 \mathrm{nM} / \mathrm{mg}$ ) (Fig. $5 \mathrm{~F}$ ). After RNAi of caspase-9, the caspase-3 activity was significantly reduced compared to that in untransfected HDPSCs $(\mathrm{P}<0.01$, one-way ANOVAs and Tukey's post hoc tests) and that in HDPSCs transfected with empty vector $(\mathrm{P}<0.01$, one-way 

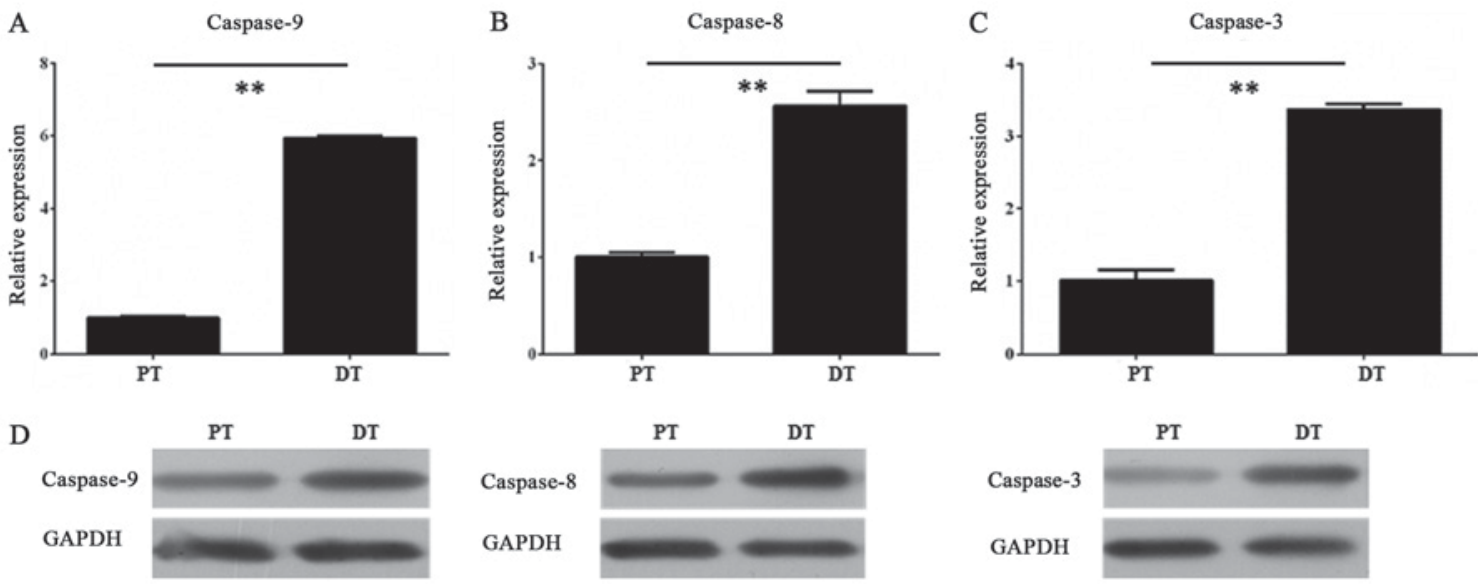

Figure 3. Expression of caspase-9, caspase-8 and caspase-3 in human dental pulp stem cells (HDPSCs). The (A-C) RT-qPCR and (D) western blotting results of (A) caspase-9, (B) caspase-8 and (C) caspase-3 in HDPSCs from adult permanent teeth (PT) and deciduous teeth (DT). Data represent the mean \pm standard deviation $(n=3)$. Statistical significance was determined with an unpaired Student's t-tests. $P<0.05$ was regarded as statistically significant $\left({ }^{* *} \mathrm{P}<0.01\right)$.

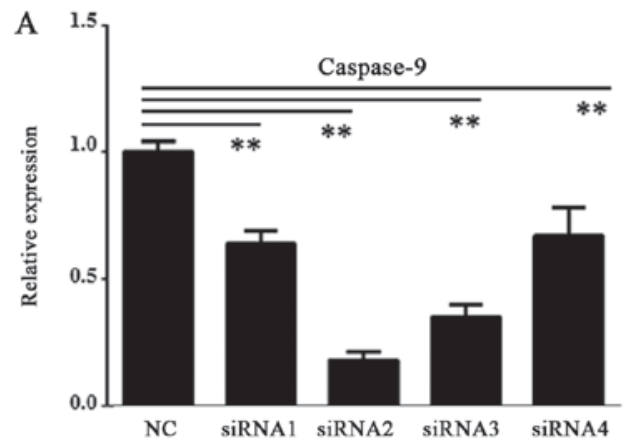

$\mathrm{C}$
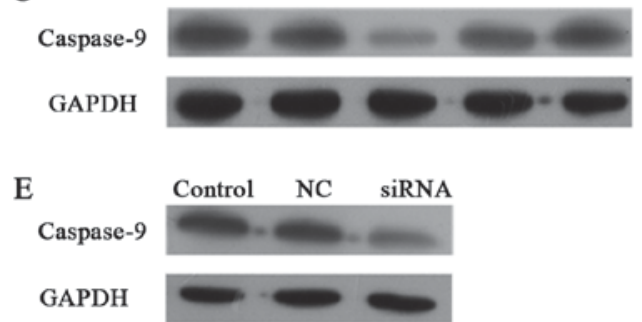

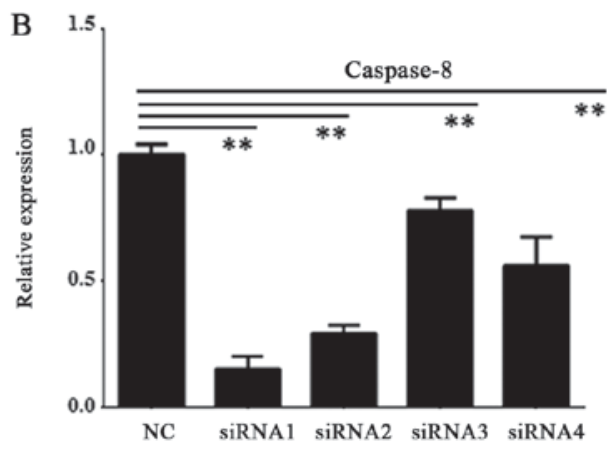

$\mathrm{D}$

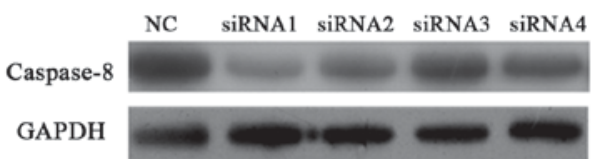

$\mathrm{F}$

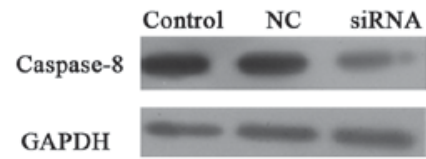

Figure 4. The RT-qPCR and western blotting results of caspase-8 and caspase-9 RNAi human dental pulp stem cells (HDPSCs) from deciduous teeth. The (A and B) RT-qPCR and (C and D) western blotting results of both caspase-9 and caspase-8 in HDPSCs transfected with siRNAs, respectively; (E and F) the western blotting results of (E) caspase-9 and (F) caspase-8 in HDPSCs transfected with best siRNAs, respectively. Data represent the mean \pm standard deviation $(\mathrm{n}=3)$. Statistical significance was determined with an one-way ANOVA test. $\mathrm{P}<0.05$ was regarded as statistically significant $\left({ }^{* * *} \mathrm{P}<0.01\right)$.

ANOVAs and Tukey's post hoc tests). By contrast, a different pattern was observed after caspase-8 RNAi treatment. There were no significant differences of caspase-3 activity among the RNAi group, untransfected HDPSCs ( $P>0.05$, one-way ANOVAs and Tukey's post hoc tests) and the HDPSCs transfected with empty vector ( $\mathrm{P}>0.05$, one-way ANOVAs and Tukey's post hoc tests) (Fig. 5F). Consistent with caspase-3 activity, the expression of caspase-3 also exhibited significant differences between the caspase-9 RNAi group and untransfected HDPSCs $(\mathrm{P}<0.01$, one-way ANOVAs and Tukey's post hoc tests) or HDPSCs transfected with empty vector $(\mathrm{P}<0.01$, one-way ANOVAs and Tukey's post hoc tests) (Fig. 5G). There were also no significant differences of caspase-3 expression among the caspase- 8 RNAi group, untransfected HDPSCs ( $\mathrm{P}>0.05$, one-way ANOVAs and Tukey's post hoc tests) and the HDPSCs transfected with empty vector ( $\mathrm{P}>0.05$, one-way ANOVAs and Tukey's post hoc tests) (Fig. 5G). Therefore, caspase-3 activity was positively correlated to its expression levels (Fig. 5H). There was a significantly positive correlation between caspase-3 expression and activity with a Pearson value of $0.9994(\mathrm{P}<0.05)$.

\section{Discussion}

Up to date, there are only a few studies about HDPSCs and apoptosis (7). According to a previous review (10), mitochondrial Cytochrome $c$ played dual roles in controlling cellular energetic metabolism and apoptosis. In mammalian cells, a major caspase-activated apoptosis pathway is initiated by Cytochrome $c$ releasing from mitochondria, which can induce 

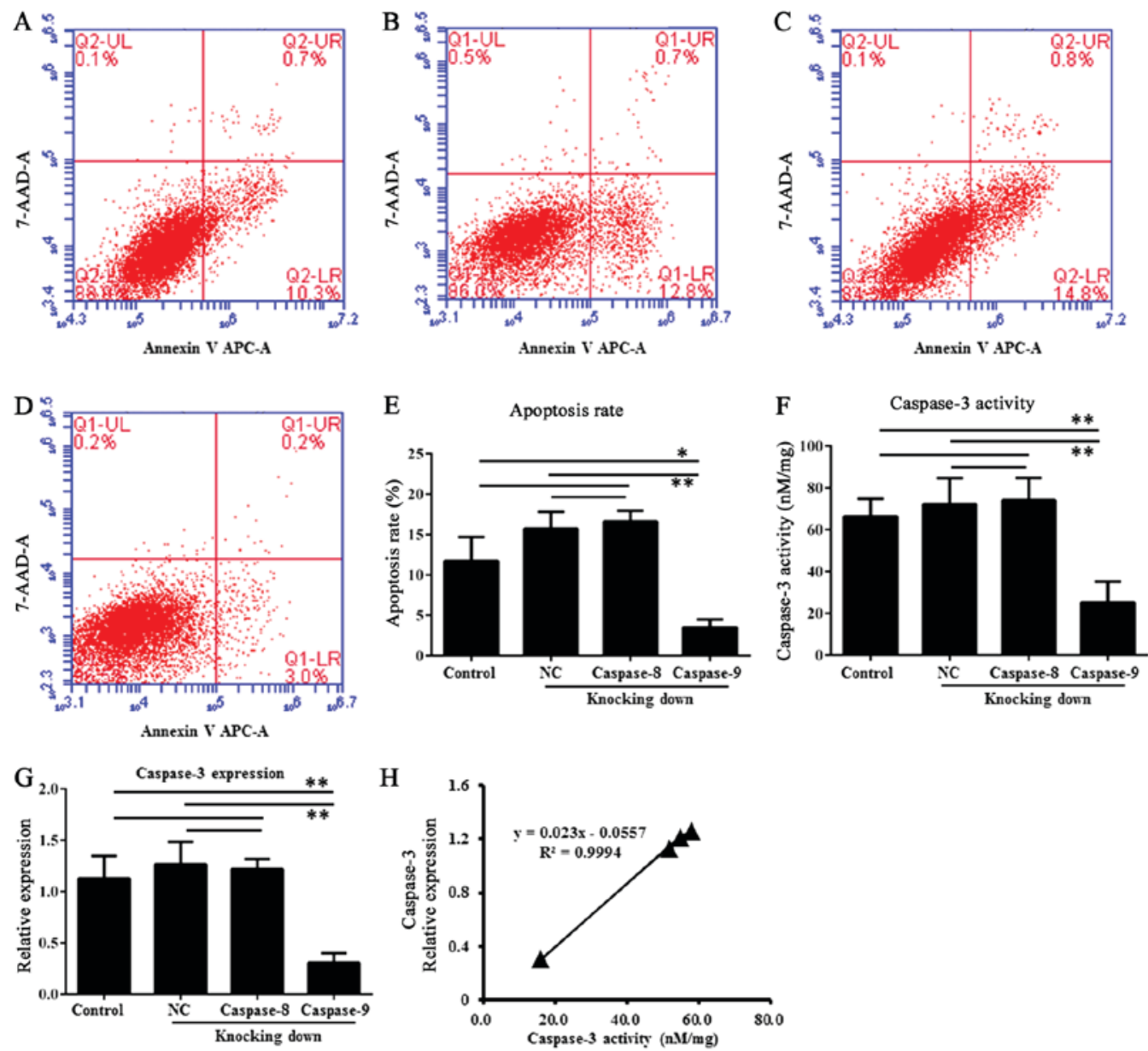

Figure 5. The apoptosis assays, caspase-3 expression and activity assays of caspase-8 and caspase-9 RNAi human dental pulp stem cells (HDPSCs) from deciduous teeth. Annexin V-FITC apoptosis assays of (A) the untransfected HDPSCs, (B) the HDPSCs transfected with empty vector, siRNAs of (C) caspase- 8 and (D) caspase-9; (E) Apoptosis rates in these four groups of HDPSCs from deciduous teeth; (F and G) The caspase-3 activity and expression assays of the untransfected HDPSCs, the HDPSCs transfected with empty vector, siRNAs of caspase-8 and caspase-9; (H) The correlation of caspase-3 expression and activity in these four groups of cells. Data represent the mean \pm standard deviation $(n=3)$. Statistical significance was determined with an one-way ANOVA test. $\mathrm{P}<0.05$ was regarded as statistically significant $\left({ }^{*} \mathrm{P}<0.05,{ }^{* *} \mathrm{P}<0.01\right)$.

caspase activation and subsequent cell death (17). The relationship of mitochondria and apoptosis have been well studied in various species, such as yeast, nematode, fly, mouse and human (18) showing that it is a common mechanism in all these species. The apoptosis of odontoclasts during physiological root resorption of human deciduous teeth was discovered, showing the presence of apoptosis in human deciduous teeth (19). In present study, the expression of Cytochrome $c$ confirmed the possibility of Cytochrome $c$-mediated apoptosis pathway in HDPSCs from both deciduous teeth and adult permanent teeth.

The mitochondrial mechanisms of apoptosis were summarized in prior reviews $(9,11,20)$. The extrinsic and intrinsic pathways require activation of caspase- 8 and caspase- 9 , respectively, which then activate caspase-3, forming two major pathways of mitochondrial apoptosis (9-11). The expression of caspase- 9 , caspase- 3 and caspase- 8 in the HDPSCs from deciduous teeth and adult permanent teeth supported the existence of apoptosis pathway. Moreover, higher expression level of all three genes in the HDPSCs from deciduous teeth might indicate higher possibility of apoptosis pathways comparing with the HDPSCs from adult permanent teeth, though it needs further confirmation. The higher expression of caspase-3 in HDPSCs from deciduous teeth was consistent with previous study, while caspase- 8 expression show some differences (21). The expression difference of caspase- 8 might be due to the examined materials we used in this study instead of deciduous teeth and adult permanent teeth.

HDPSCs were thought to originate from migrating neural crest cells during development, because they reside predominantly within the perivascular niche of dental pulp (22). According to Zhao et al, ADAM28 was involved in the proliferation, differentiation, and apoptosis of HDPSCs (8), however, whether other pathways were involved in apoptosis of HDPSCs remains unknown. As mentioned above, the extrinsic and intrinsic pathways were typical in apoptosis, which needs activation of caspase- 9 , caspase- 8 and caspase-3 (9-11). The knock down of caspase-9 induced reduction of apoptosis in the HDPSCs from deciduous teeth, while that of caspase- 8 did not. This result suggested that the caspase-9-mediated pathway may be more important in apoptosis than caspase- 8 -mediated pathway in HDPSCs.

In previous reviews (9-11), caspase- 3 was activated by caspase- 8 or caspase- 9 , though whether the expression of caspase- 3 was regulated by caspase- 8 or caspase- 9 remians 
unclear. The HDPSCs transfected with caspase- 8 siRNA1 and caspase-9 siRNA2 showed that knocking down of caspase-9 instead of caspase- 8 induced significant reduction of caspase- 3 expression and activity. Therefore, caspase-3 expression and activity should be regulated by caspase- 9 in HDPSCs. This line of evidence also supported that caspase- 9 followed by activated caspase-3 were involved in HDPSCs cell apoptosis. Additionally, caspase- 8 did not regulate caspase- 3 expression in HDPSCs from deciduous teeth. Whether caspase- 8 regulate caspase-3 expression in other HDPSCs or other types of cells need further study.

As we known, HDPSCs were capable to form dentin-pulp complex-like structures or a woven bone-like structure in vivo, therefore, they had great potential for tissue engineering $(4,23,24)$. Our study here uncovered the apoptosis pathway in HDPSCs, which may provide theoretical framework for further application.

\section{Acknowledgements}

The authors would like to thank Guangzhou Genedenovo Biotechnology Co., Ltd. for their technical support and revision of manuscript.

\section{Funding}

This study was supported by a grant from Guangzhou City Science and Technology Plan (grant no. 1563000340).

\section{Availability of data and materials}

The datasets used and/or analyzed during the current study are available from the corresponding author on reasonable request.

\section{Authors' contributions}

HQ conceived and designed the study, and wrote the paper. QH, YXC, MWY, JXF and QL collected the clinical samples, performed the experiments, analyzed the data, interpreted the results, and prepared figures for the paper. All authors read and approved the final manuscript.

\section{Ethics approval and consent to participate}

All procedures used in this study conformed to the tenets of the Declaration of Helsinki. The Ethics Committee guidelines of Stomatological Hospital, Southern Medical University approved the protocols used. Informed consent was obtained from all participants.

\section{Consent for publication}

Not applicable.

\section{Competing interests}

The authors declare that they have no competing interests.

\section{References}

1. Miura M, Gronthos S, Zhao M, Lu B, Fisher LW, Robey PG and Shi S: SHED: Stem cells from human exfoliated deciduous teeth. Proc Natl Acad Sci USA 100: 5807-5812, 2003.

2. Gronthos S, Brahim J, Li W, Fisher LW, Cherman N, Boyde A, DenBesten P, Robey PG and Shi S: Stem cell properties of human dental pulp stem cells. J Dent Res 81: 531-535, 2002.

3. Muthna D, Soukup T, Vavrova J, Mokry J, Cmielova J, Visek B, Jiroutova A, Havelek R, Suchanek J, Filip S, et al: Irradiation of adult human dental pulp stem cells provokes activation of p53, cell cycle arrest, and senescence but not apoptosis. Stem Cells Dev 19: 1855-1862, 2010.

4. Gronthos S, Mankani M, Brahim J, Robey PG and Shi S: Postnatal human dental pulp stem cells (DPSCs) in vitro and in vivo. Proc Natl Acad Sci USA 97: 13625-13630, 2000.

5. Bakopoulou A and About I: Stem cells of dental origin: Current research trends and key milestones towards clinical application. Stem Cells Int 2016: 4209891, 2016.

6. Kim S, Shin SJ, Song Y and Kim E: In vivo experiments with dental pulp stem cells for pulp-dentin complex regeneration. Mediators Inflamm 2015: 409347, 2015.

7. Kobayashi C, Yaegaki K, Calenic B, Ishkitiev N, Imai T, Ii H, Aoyama I, Kobayashi H, Izumi Y and Haapasalo M: Hydrogen sulfide causes apoptosis in human pulp stem cells. J Endod 37: 479-484, 2011.

8. Zhao Z, Liu H and Wang D: ADAM28 manipulates proliferation, differentiation, and apoptosis of human dental pulp stem cells. J Endod 37: 332-339, 2011.

9. Elmore S: Apoptosis: A review of programmed cell death. Toxicol Pathol 35: 495-516, 2007.

10. Cai J, Yang J and Jones DP: Mitochondrial control of apoptosis: The role of cytochrome c. Biochim Biophys Acta 1366: 139-149, 1998.

11. Circu ML and Aw TY: Reactive oxygen species, cellular redox systems, and apoptosis. Free Radic Biol Med 48: 749-762, 2010.

12. Hannon GJ: RNA interference. Nature 418: 244-251, 2002.

13. Sui G, Soohoo C, Affar el B, Gay F, Shi Y, Forrester WC and Shi Y: A DNA vector-based RNAi technology to suppress gene expression in mammalian cells. Proc Natl Acad Sci USA 99: 5515-5520, 2002.

14. Livak KJ and Schmittgen TD: Analysis of relative gene expression data using real-time quantitative PCR and the 2(-Delta Delta C(T)) method. Methods 25: 402-408, 2001.

15. Altschul SF, Madden TL, Schäffer AA, Zhang J, Zhang Z, Miller W and Lipman DJ: Gapped BLAST and PSI-BLAST: A new generation of protein database search programs. Nucleic Acids Res 25: 3389-3402, 1997.

16. Nakamura S, Yamada Y, Katagiri W, Sugito T, Ito K and Ueda M: Stem cell proliferation pathways comparison between human exfoliated deciduous teeth and dental pulp stem cells by gene expression profile from promising dental pulp. J Endod 35: 1536-1542, 2009.

17. Jiang $X$ and Wang X: Cytochrome C-mediated apoptosis. Annu Rev Biochem 73: 87-106, 2004

18. Karbowski M and Youle RJ: Dynamics of mitochondrial morphology in healthy cells and during apoptosis. Cell Death Differ 10: 870-880, 2003.

19. Domon T, Taniguchi Y, Inoue K, Ushijima N, Taishi Y, Hiramatsu A, Wakita M and Yoshida S: Apoptosis of odontoclasts under physiological root resorption of human deciduous teeth. Cell Tissue Res 331: 423-433, 2008.

20. Polster BM and Fiskum G: Mitochondrial mechanisms of neural cell apoptosis. J Neurochem 90: 1281-1289, 2004.

21. Rodrigues LV, Del Puerto HL, Brant JM, Leite RC and Vasconcelos AC: Caspase-3/caspase- 8 , bax and bcl2 in pulps of human primary teeth with physiological root resorption. Int J Paediatr Dent 22: 52-59, 2012.

22. Stokowski A, Shi S, Sun T, Bartold PM, Koblar SA and Gronthos S: EphB/ephrin-B interaction mediates adult stem cell attachment, spreading, and migration: Implications for dental tissue repair. Stem Cells 25: 156-164, 2007.

23. About I, Bottero MJ, de Denato P, Camps J, Franquin JC and Mitsiadis TA: Human dentin production in vitro. Exp Cell Res 258: 33-41, 2000.

24. d'Aquino R, De Rosa A, Laino G, Caruso F, Guida L, Rullo R, Checchi V, Laino L, Tirino V and Papaccio G: Human dental pulp stem cells: From biology to clinical applications. J Exp Zool B Mol Dev Evol 312B: 408-415, 2009. 